Open Access

\title{
Approaches and impact of non-academic research capacity strengthening training models in sub-Saharan Africa: a systematic review
}

Lambert Mugabo ${ }^{1,2^{*}}$, Dominique Rouleau', Jackline Odhiambo ${ }^{1}$, Marie Paul Nisingizwe ${ }^{1}$, Cheryl Amoroso ${ }^{1}$, Peter Barebwanuwe ${ }^{1}$, Christine Warugaba' ${ }^{1}$ Lameck Habumugisha ${ }^{1}$ and Bethany L. Hedt-Gauthier ${ }^{1,3}$

\begin{abstract}
Background: Research is essential to identify and prioritize health needs and to develop appropriate strategies to improve health outcomes. In the last decade, non-academic research capacity strengthening trainings in sub-Saharan Africa, coupled with developing research infrastructure and the provision of individual mentorship support, has been used to build health worker skills. The objectives of this review are to describe different training approaches to research capacity strengthening in sub-Saharan Africa outside academic programs, assess methods used to evaluate research capacity strengthening activities, and learn about the challenges facing research capacity strengthening and the strategies/innovations required to overcome them.

Methodology: The PubMed database was searched using nine search terms and articles were included if 1) they explicitly described research capacity strengthening training activities, including information on program duration, target audience, immediate program outputs and outcomes; 2) all or part of the training program took place in sub-Saharan African countries; 3) the training activities were not a formal academic program; 4) papers were published between 2000 and 2013; and 5) both abstract and full paper were available in English.

Results: The search resulted in 495 articles, of which 450 were retained; 14 papers met all inclusion criteria and were included and analysed. In total, 4136 people were trained, of which 2939 were from Africa. Of the 14 included papers, six fell in the category of short-term evaluation period and eight in the long-term evaluation period. Conduct of evaluations and use of evaluation frameworks varied between short and long term models and some trainings were not evaluated. Evaluation methods included tests, surveys, interviews, and systems approach matrix.

Conclusions: Research capacity strengthening activities in sub-Saharan Africa outside of academic settings provide important contributions to developing in-country capacity to participate in and lead research. Institutional support, increased funds, and dedicated time for research activities are critical factors that lead to the development of successful programs. Further, knowledge sharing through scientific articles with sufficient detail is needed to enable replication of successful models in other settings.
\end{abstract}

Keywords: Capacity strengthening, Capacity building, Outcomes, sub-Saharan Africa, Systematic review

\footnotetext{
* Correspondence: lambertmugabo@gmail.com

${ }^{1}$ Partners In Health-Inshuti Mu Buzima, P.O. Box 3432, Kigali, Rwanda

${ }^{2}$ Global Health Corps, One Penn Plaza, Suite 6271, New York, NY 10119, USA

Full list of author information is available at the end of the article
} 


\section{Background}

High quality research is essential to identify and prioritize health needs and to develop appropriate strategies to improve health outcomes [1]. However, despite the increase of publications from Africa during the past two decades [2], the representation of Africa in global research output is disproportionately low. For example, between 1997 and 2006, only 7 \% of global tuberculosis research output came from Africa despite the region having the highest tuberculosis case rates in the world [3]. In 2004, research about Africa represented less than $1 \%$ of scientific publications [4], growing gradually to $10 \%$ as of 2011 [5].

In the last decade, the international call for developing research capacity in sub-Saharan Africa has grown $[4,5]$. Opportunities to support individuals pursuing academic studies and fellowships at academic institutions have increased $[6,7]$. However, there are several limitations to academic programs as the sole means for capacity strengthening in sub-Saharan Africa - they can be long to complete and expensive and present a potential risk of drawing national researchers from program settings into academia, especially if no strong partnerships exist between academia and local programs [3]. Further, academic research tends to miss operational perspectives from programs [3]. To overcome these limitations and as a complement to these academic programs, local organizations/institutions across Africa, often in partnership with institutions from developed countries, have implemented short trainings targeting specific research competencies of health program staff. The term 'nonacademic' is used throughout this paper to refer to training programs that do not lead to formal academic qualifications, although they may use academic training staff and/or infrastructure.

Strengthening research capacity in non-academic settings encompasses a variety of activities, including trainings to support individuals to acquire research skills in addition to developing research infrastructure at an institutional level, creating research partnerships/networks, and providing individual support and mentorship [8]. In this paper, we focus specifically on the training activities in the research capacity strengthening programs. The goals, approaches, target audience, and effectiveness of the skill-specific research trainings in sub-Saharan Africa vary widely. However, there are few peer-reviewed published descriptions of these activities to support the replication or adaptation of such programs in other locations. The objectives of this systematic review are therefore 1) to describe the different approaches to research capacity strengthening in subSaharan Africa beyond academic programs, 2) to assess methods used to evaluate research capacity strengthening activities and summarize their results, and 3) to learn about challenges to research capacity strengthening and strategies/innovations to overcome those challenges. This review will contribute to research capacity strengthening efforts by providing insights from different approaches that could be applied to other locations and to encourage more complete reporting of such initiatives.

\section{Methods}

\section{Identification of data sources}

The PubMed database was searched by the principal investigator (LM) for articles describing research capacity strengthening training activities in Africa. The following search terms were used (illustrated in Fig. 1): words that indicate an increase in competency ("building", "development", "strengthening", and "training") combined with "capacity" as well as the terms "Africa" and "health" and "research". Further search criteria were 1) papers published between 2000 and 2013 and 2) both abstract and full paper available in English. The results were saved into a Mendeley library.

\section{Study selection}

The titles and abstracts were reviewed by the principal investigator (LM) to ensure they met the following inclusion criteria: 1) research capacity strengthening training activities are explicitly described, including information on program duration, target audience, and immediate program outputs and outcomes, 2) all or part of the training program took place in sub-Saharan African countries, and 3) the training activities are not a formal academic program. When all criteria were met, or more information was needed, articles were retained for full text review (Fig. 2). Articles were also assessed after full text review and dropped if not meeting all eligibility criteria. Articles not captured in the original search were added, either because they were known to the authors or were identified through a snowballing process of reviewing the reference list of retained articles.

\section{Data extraction and analysis}

Two independent reviewers extracted data from the full text articles, which was captured in a three-part data collection form. The form was developed based on the research team's experience in conducting research strengthening activities and adapted based on themes that emerged during the review of articles. The first part covered program description information, including name of the program, program duration, target audience, objectives of the training, frequency of the training, qualification of the trainers, resources required for the training, and where the training took place. The second part used Cooke's evaluation framework to assess the effectiveness of the trainings [9]. Cooke's framework was chosen because it comprehensively describes the indicators 


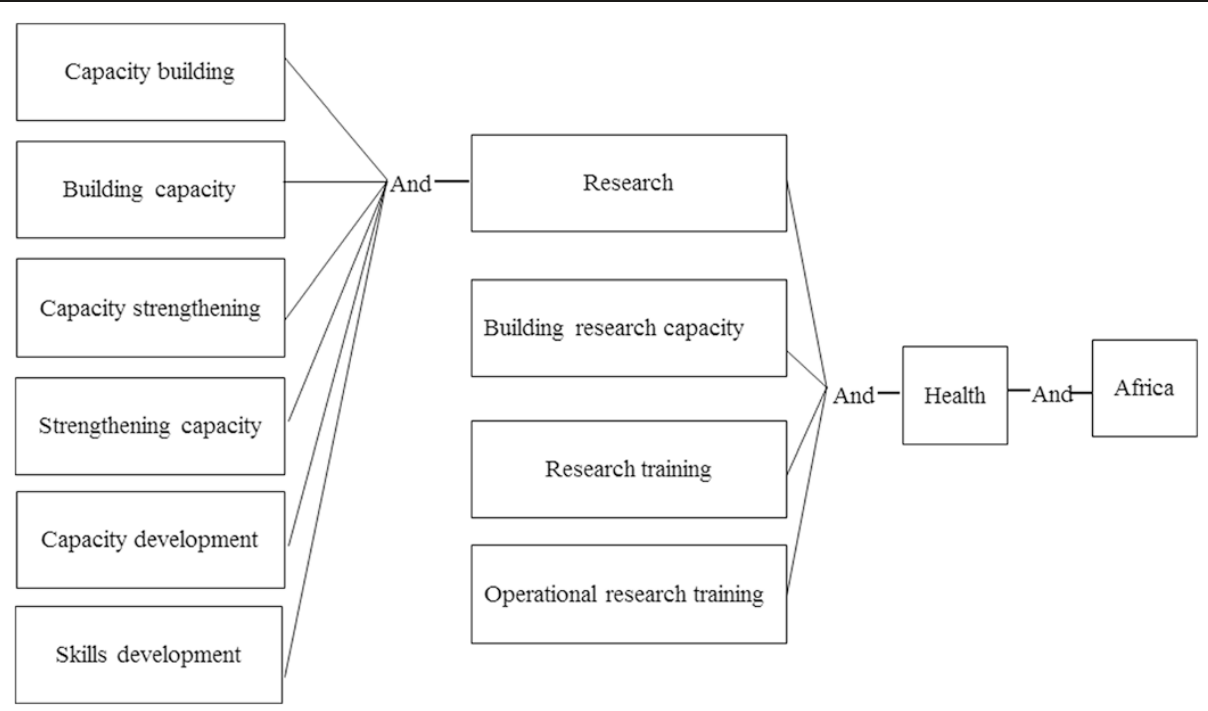

Fig. 1 Search terms for systematic review

for individual training in research capacity building based on six principles: research skills, practice implications, partnerships, dissemination, infrastructure, and sustainability. We grouped the trainings based on their evaluation period. The short term evaluation period was defined as evaluations conducted up to 18 months after the training. The long-term evaluation period was any period greater than 18 months.

Finally, data were extracted on challenges faced, innovations used, and recommendations proposed for future programs. Data extractions from both reviewers were entered into a Microsoft Access database and compared for consistency. When inter-reviewer discrepancies were found, they were resolved by a third party review of the paper.

\section{Results}

The search resulted in 495 articles, of which 450 were retained following the removal of duplicates (Fig. 2). Based on abstract review, 24 articles were classified as potentially relevant, 425 were dropped, and one abstract was not available. Full text review of the 24 articles yielded 11 relevant texts. The same training program was presented in two of these articles and so only the more recent and relevant publication was retained. Four additional articles were identified through the snowball process, resulting in 14 relevant articles in total.

Description of the research capacity strengthening programs The 14 research capacity strengthening trainings described in these papers include four which started in the

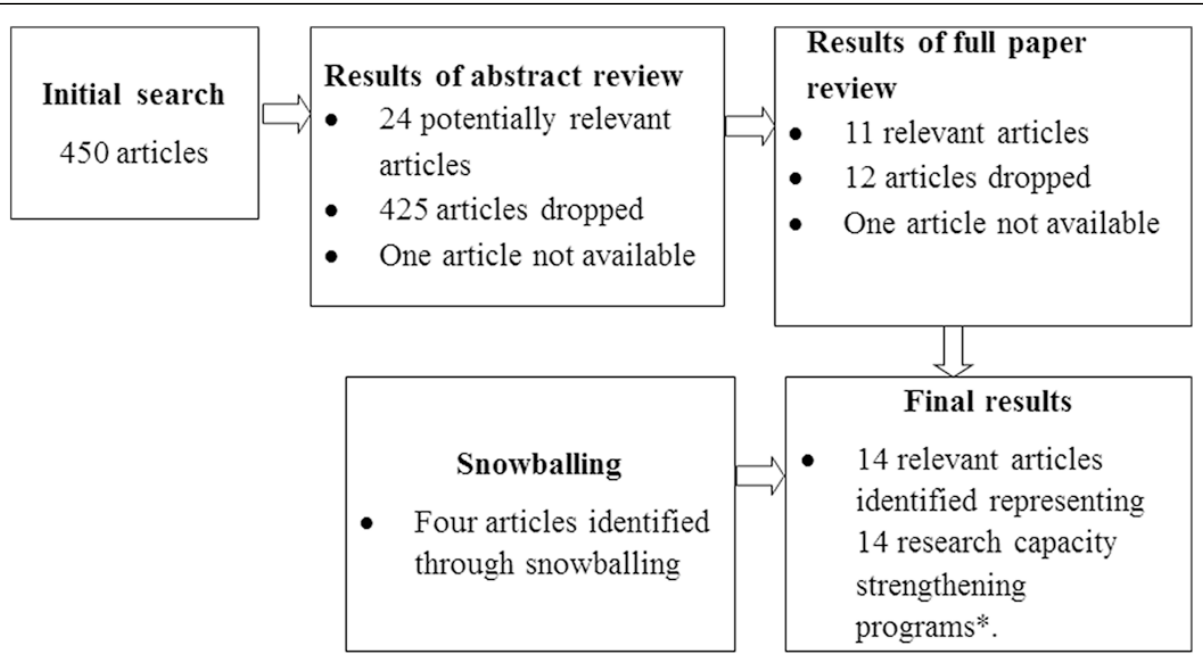

Fig. 2 Search and selection process in the review on research capacity strengthening in sub-Saharan Africa 
1990s [10-13] and the rest from 2000 or later [14-21] (Table 1). Most programs took place in the southern African region $[10,12,13,20]$, followed by Uganda [16, 18]. Malawi [21], Nigeria [14], Cameroon [17], and the Democratic Republic of Congo [19] were also represented. For two trainings, some of the training activities took place in the United States of America [15] or Europe [11]. Most of the training activities were implemented multiple times $[10-16,20,22]$.

\section{Research competencies covered}

Of the 14 training programs identified, eight covered multiple competencies $[10-13,16,18,21,23]$, while six focused on a single research competency $[14,15,17,19$, $20,22]$. The most covered competencies included research ethics $[10,11,14,15,18,19,22]$, research methods $[10-12,15,18]$, data collection $[10-13,16,18$, 21], data analysis $[10-13,16,17,20,21,23]$, research protocol/research design $[10-13,16,21,23]$, and writing a report or manuscript $[10-13,16,21,23]$. Of six training programs focusing on a single research competency, four focused on research ethics [14, 15, 19, 22] and one focused on systematic reviews [17], whereas the other one targeted data management and analysis skills [20].

\section{Target audience and trainers}

In total, 4136 people were trained of which at least 2939 were from Africa. Trainees in these programs were of very different backgrounds and qualifications. Participants included clinical staff, health officers and managers working within health programs, university students and faculty, and experienced researchers. Generally, participants were selected based on their potential to influence health systems and management processes, ability to conduct research activities, and their involvement or expertise in the field. Only two training programs had a rigorous selection process whereby criteria, such as years of experience in research ethics, number of publications, institutional support, and personal commitment, were considered $[15,23]$. For 11 of the 14 programs, participants were from the country where the training took place. However, three of the programs required out-of-country travel of the participants to the training site. For these, one was in sub-Saharan Africa [10] and two in Europe or USA $[15,23]$.

Specific details about the qualifications of trainers were not reported. Most of them were individuals with experience and expertise in the area of interest, either based in the country or brought in through a partnership as an international expert. They included faculty from universities, researchers, and practitioners in a given field.

\section{Structure, duration of the trainings and follow-up}

Structure and duration of research strengthening activities outside academic settings vary widely. Five of 14 training programs $[10,14,17,19,20]$ only featured faceto-face sessions conducted over a short period of time; mostly less than a week. Seven training programs featured both face-to-face sessions and practicums. Of these, four had face-to-face sessions spread over a longer period with intervals of field activities taking place in between $[13,16,23]$. Face-to-face sessions took at least 3 weeks, whilst one training program featured short classes of $21 / 2$ days [21]. Three training programs mixing face-to-face sessions with practicums conducted the practicum after the face-to-face sessions. Two had 6 days of classes [12, 18], whilst another one had a longer period of face-to-face classes [15] and practicum ranges between 1 [18] and 12 months [15]. Further, one training program had five courses conducted over 5 days each, structured as a ladder [11], where success at a lower level determined who moved up to the next. Finally, one training program was web-based [22], taking 100 days to complete. Seven training programs provided follow-up to their trainees $[11,12,15,16,18,21,23]$ in terms of ongoing mentorship, onsite technical assistance, and supervisory visits.

\section{Evaluation}

Of the 14 training programs, six fell in the category of short-term evaluation period [14, 17-19, 21, 22] and eight in the long-term evaluation period $[10-13,15,16$, $20,23]$. For the training programs with a short-term evaluation period, one was not evaluated [19] and only one $(16.7 \%)$ used a recognized framework for evaluation [18]. These training programs used quantitative evaluation methods, mainly surveys and tests. Of the training programs with long-term evaluation periods, $37.5 \%$ $(\mathrm{n}=3)$ used a framework $[10,15,16]$. All of these training programs were evaluated, using quantitative or qualitative methods including interviews, surveys, and systems approach framework.

\section{Training programs with short term evaluation period}

All training programs reported an increase in research knowledge and skills (100\%; Table 2). More than half of the training programs (50-67.7\%), reported the involvement of practitioner and program staff in the training, the relevance or use of training related research in practice, and the existence of inter-professional linkages. None of these training programs, however, reported on or used impactful dissemination (publication, conferences, workshop presentations, changes in policy and practice) as a key principle in research capacity strengthening. Further, there was no information about conduct of research after training, patient centred outcome 
Table 1 Characteristics of approaches in the review of research capacity strengthening activities in sub-Saharan Africa

\begin{tabular}{|c|c|c|c|c|c|c|c|}
\hline Reference & $\begin{array}{l}\text { Location of the } \\
\text { training }\end{array}$ & $\begin{array}{l}\text { Training goal and specific } \\
\text { competencies }\end{array}$ & Target trainees & Faculty/trainers & $\begin{array}{l}\text { Structure, duration of the } \\
\text { training activities, and } \\
\text { frequency of offering }\end{array}$ & Funding and partnership & $\begin{array}{l}\text { Technical support/ } \\
\text { follow-up during the } \\
\text { program }\end{array}$ \\
\hline $\begin{array}{l}\text { Adams et al. } \\
2003[10]\end{array}$ & South Africa & $\begin{array}{l}\text { Goal: Provide skills for } \\
\text { health service evaluation } \\
\text { Competencies: Research } \\
\text { ethics, research methods, } \\
\text { data capture and analysis, } \\
\text { research protocol and } \\
\text { report writing }\end{array}$ & $\begin{array}{l}300 \text { trainees, all African: } \\
\text { Health service middle } \\
\text { managers and MSc } \\
\text { students within the } \\
\text { country }\end{array}$ & In-country based faculty & $\begin{array}{l}\text { Training activities lasted } \\
2 \text { weeks and offered } 13 \\
\text { times during 1992-2001 }\end{array}$ & $\begin{array}{l}\text { Total funds: Not reported } \\
\text { (materials expenses } \\
\text { mentioned) Funding } \\
\text { source and partnerships: } \\
\text { Not reported }\end{array}$ & No \\
\hline $\begin{array}{l}\text { Ajuwon and } \\
\text { Kass } 2008 \text { [14] }\end{array}$ & Nigeria & $\begin{array}{l}\text { Goal: To develop the } \\
\text { capacity of academic staff } \\
\text { to conduct ethically } \\
\text { acceptable research } \\
\text { involving human } \\
\text { population } \\
\text { Competencies: Research } \\
\text { ethics }\end{array}$ & $\begin{array}{l}133 \text { trainees, all African: } \\
\text { Clinical staff from College } \\
\text { of Medicine and } \\
\text { researchers from NGOs } \\
\text { and IRB }\end{array}$ & $\begin{array}{l}\text { Locally based seven } \\
\text { resource persons with } \\
\text { experience }\end{array}$ & $\begin{array}{l}\text { Training activities lasted } \\
21 \text { hours spread over } \\
3 \text { days and offered three } \\
\text { times during 2003-2004 }\end{array}$ & $\begin{array}{l}\text { Total funds: Not reported } \\
\text { (materials expenses } \\
\text { mentioned) Funding } \\
\text { source and partnerships: } \\
\text { NIH, Wellcome Trust, } \\
\text { Fogarty International } \\
\text { Center }\end{array}$ & No \\
\hline $\begin{array}{l}\text { Ali et al. } \\
2012[15]\end{array}$ & $\begin{array}{l}\text { Blended between } \\
\text { USA and the } \\
\text { country of origin }\end{array}$ & $\begin{array}{l}\text { Goal: Training on research } \\
\text { ethics to health } \\
\text { professionals. } \\
\text { Competencies: Ethics and } \\
\text { research methods }\end{array}$ & $\begin{array}{l}28 \text { trainees, all African: } \\
\text { Researchers mainly from } \\
\text { Eastern Africa, most of } \\
\text { which had graduate } \\
\text { degrees with research } \\
\text { experience, health } \\
\text { professionals, ethics } \\
\text { committee members, } \\
\text { journalists and scientists }\end{array}$ & $\begin{array}{l}\text { Associate faculty from } \\
\mathrm{JHU} \text {, the } \mathrm{NIH} \text {, associated } \\
\text { research ethics programs, } \\
\text { and African professionals }\end{array}$ & $\begin{array}{l}\text { Program lasted } 1 \text { year, } \\
6 \text { months of courses and } \\
\text { seminars, IRB involvement } \\
\text { and development of field } \\
\text { project and } 6 \text { months for } \\
\text { practicum and was } \\
\text { offered multiple times } \\
\text { during 2001-2009 }\end{array}$ & $\begin{array}{l}\text { Total funds: Not reported } \\
\text { (materials, flight expenses } \\
\text { mentioned) Funding } \\
\text { source and partnerships: } \\
\text { NIH }\end{array}$ & $\begin{array}{l}\text { Continuous } \\
\text { mentorship from JHU } \\
\text { and African faculty, } \\
\text { biannual reunion } \\
\text { meeting of alumni } \\
\text { and faculty for } \\
\text { networking and } \\
\text { exchanging ideas }\end{array}$ \\
\hline $\begin{array}{l}\text { Matovu et al. } \\
2013[16]\end{array}$ & Uganda & $\begin{array}{l}\text { Goal: Strengthen the } \\
\text { capacity of M\&E and } \\
\text { continuous quality } \\
\text { improvement using work- } \\
\text { based training model } \\
\text { Competencies: Data col- } \\
\text { lection, data analysis, pro- } \\
\text { ject proposal, report } \\
\text { writing and M\&E }\end{array}$ & $\begin{array}{l}143 \text { trainees, all African: } \\
\text { Mid-and senior-level } \\
\text { managers, coordinators } \\
\text { and supervisors within } \\
\text { the country }\end{array}$ & $\begin{array}{l}\text { MakSPH faculty and } \\
\text { external facilitators }\end{array}$ & $\begin{array}{l}\text { Training activities lasted } \\
5 \text { weeks of face-to-face } \\
\text { sessions and } 6 \text { months of } \\
\text { field activities between } \\
\text { 2nd and 3rd modules } \\
\text { and was offered multiple } \\
\text { times during 2008-2011 }\end{array}$ & $\begin{array}{l}\text { Total funds: } \$ 2500 \text { for } \\
\text { project implementation } \\
\text { Funding source and } \\
\text { partnerships: CDC }\end{array}$ & $\begin{array}{l}\text { Ongoing technical } \\
\text { support from an } \\
\text { academic mentor } \\
\text { over the program }\end{array}$ \\
\hline $\begin{array}{l}\text { Mbuagbaw } \\
\text { et al. } 2011 \text { [17] }\end{array}$ & Cameroon & $\begin{array}{l}\text { Goal: Training on how to } \\
\text { initiate and complete } \\
\text { systematic reviews } \\
\text { Competencies: Design, } \\
\text { analysis and } \\
\text { interpretation of } \\
\text { systematic review and } \\
\text { meta-analysis }\end{array}$ & $\begin{array}{l}15 \text { trainees, all African: } \\
\text { University lecturers and } \\
\text { researchers within the } \\
\text { country }\end{array}$ & $\begin{array}{l}\text { Cochrane Review authors } \\
\text { and researchers from } \\
\text { Africa and Chile }\end{array}$ & $\begin{array}{l}\text { Training activities lasted } \\
4 \text { days of face-to-face ses- } \\
\text { sions and was offered } \\
\text { once in } 2011\end{array}$ & $\begin{array}{l}\text { Total funds: Not reported } \\
\text { Funding source and } \\
\text { partnerships: Cochrane } \\
\text { Collaboration, South } \\
\text { African Medical Research } \\
\text { Council, Yaunde Central } \\
\text { Hospital, and Global } \\
\text { Health Research Initiative }\end{array}$ & No \\
\hline $\begin{array}{l}\text { Njie-Carr et al. } \\
2012 \text { [18] }\end{array}$ & Uganda & $\begin{array}{l}\text { Goal: Research capacity } \\
\text { building to assess } \\
\text { implementation of } \\
\text { mobile service for HIV } \\
\text { intervention }\end{array}$ & $\begin{array}{l}14 \text { trainees, all African: } \\
\text { Employees and } \\
\text { volunteers at Reach Out, } \\
\text { a large HIV/AIDS care and }\end{array}$ & $\begin{array}{l}\text { Five authors in total from } \\
\text { fields of medicine, } \\
\text { nursing, psychology, } \\
\text { biology, and public and } \\
\text { international health }\end{array}$ & $\begin{array}{l}\text { Training activities lasted } \\
6 \text { days of intensive } \\
\text { didactic training and } \\
4 \text { weeks of field activities } \\
\text { and offered once in } 2010\end{array}$ & $\begin{array}{l}\text { Total funds: Not reported } \\
\text { (software fees mentioned) } \\
\text { Funding source and } \\
\text { partnerships: RO, }\end{array}$ & $\begin{array}{l}\text { Continuous } \\
\text { mentorship from } \\
\text { trainers over the } \\
\text { program }\end{array}$ \\
\hline
\end{tabular}


Table 1 Characteristics of approaches in the review of research capacity strengthening activities in sub-Saharan Africa (Continued)

\begin{tabular}{|c|c|c|c|c|c|c|c|}
\hline & & $\begin{array}{l}\text { Competencies: Research } \\
\text { ethics, research methods, } \\
\text { data collection }\end{array}$ & $\begin{array}{l}\text { service program in } \\
\text { Kampala }\end{array}$ & & & $\begin{array}{l}\text { Makerere University, and } \\
\text { Johns Hopkins }\end{array}$ & \\
\hline $\begin{array}{l}\text { Tshikala et al. } \\
2012 \text { [19] }\end{array}$ & $\begin{array}{l}\text { Democratic } \\
\text { Republic } \\
\text { of Congo }\end{array}$ & $\begin{array}{l}\text { Goal: Train on research } \\
\text { bioethics through } \\
\text { ancillary care } \\
\text { Competencies: Research } \\
\text { ethics }\end{array}$ & $\begin{array}{l}30 \text { trainees, all African: } \\
\text { Members of CIBAF, faculty } \\
\text { from universities, } \\
\text { members of clinical ethics } \\
\text { committee, } \\
\text { representatives of NGOs, } \\
\text { paediatric clinics and } \\
\text { National AIDS Control } \\
\text { Program, members of } \\
\text { UNC/DRC }\end{array}$ & $\begin{array}{l}\text { Members of GIRIE, CIBAF } \\
\text { and KSPH faculty }\end{array}$ & $\begin{array}{l}\text { Training activities lasted } \\
3 \text { days of formal } \\
\text { presentations and } \\
\text { discussion and offered } \\
\text { once }\end{array}$ & $\begin{array}{l}\text { Total funds: Not reported } \\
\text { (materials expenses } \\
\text { mentioned) Funding } \\
\text { source and partnerships: } \\
\text { NIH/Fogarty International } \\
\text { Center }\end{array}$ & No \\
\hline $\begin{array}{l}\text { Williams et al. } \\
2010 \text { [20] }\end{array}$ & South Africa & $\begin{array}{l}\text { Goal: Increase research } \\
\text { training and utilization of } \\
\text { existing datasets } \\
\text { Competencies: Data } \\
\text { management and analysis }\end{array}$ & $\begin{array}{l}55 \text { trainees, } 40 \text { African: } \\
\text { Masters and PhD students } \\
\text { from Wits, CU, Brown } \\
\text { University and researchers } \\
\text { from APHRC }\end{array}$ & $\begin{array}{l}\text { CU researchers, } \\
\text { Institutional faculty from } \\
\text { Wits, CU, Brown } \\
\text { University and APHRC }\end{array}$ & $\begin{array}{l}\text { Training activities lasted } \\
3 \text { weeks of lectures, } \\
\text { guided exercises, and } \\
\text { research projects and was } \\
\text { offered three times } \\
\text { during 2006-2008 }\end{array}$ & $\begin{array}{l}\text { Total funds: Not reported } \\
\text { Funding source and } \\
\text { partnerships: Wit School } \\
\text { of Public Health, } \\
\text { University of Colorado, } \\
\text { African Population } \\
\text { Studies Research and } \\
\text { Training Program }\end{array}$ & No \\
\hline \multirow[t]{2}{*}{$\begin{array}{l}\text { Buist and Parry } \\
2013[11]\end{array}$} & \multirow[t]{2}{*}{$\begin{array}{l}\text { Multiple countries } \\
\text { in sub-Saharan } \\
\text { Africa }\end{array}$} & \multirow{2}{*}{$\begin{array}{l}\text { Goal: increasing local and } \\
\text { national research capacity } \\
\text { Competencies: Research } \\
\text { ethics, research methods, } \\
\text { data collection and } \\
\text { analysis, research protocol } \\
\text { and writing report }\end{array}$} & \multirow{2}{*}{$\begin{array}{l}1015 \text { trainees, } 112 \text { African: } \\
\text { Practicing and academic } \\
\text { physicians and public } \\
\text { health professionals } \\
\text { across Africa }\end{array}$} & \multirow{2}{*}{$\begin{array}{l}\text { Local and international } \\
\text { behavioural, } \\
\text { epidemiologic, public } \\
\text { health, and statistical } \\
\text { researchers }\end{array}$} & $\begin{array}{l}\text { Five courses structured as } \\
\text { a ladder with each one } \\
\text { lasting } 5 \text { to } 51 / 2 \text { days }\end{array}$ & \multirow{2}{*}{$\begin{array}{l}\text { Total funds: Not reported } \\
\text { (material expenses } \\
\text { mentioned) Funding } \\
\text { source and partnerships: } \\
\text { CDC, USAID, ATS }\end{array}$} & \multirow{2}{*}{$\begin{array}{l}\text { Students receive } \\
\text { mentoring following } \\
\text { the course, incentives } \\
\text { to support research } \\
\text { projects and editorial } \\
\text { assistance are } \\
\text { provided }\end{array}$} \\
\hline & & & & & $\begin{array}{l}\text { Courses involved lectures, } \\
\text { small groups to develop } \\
\text { protocol, and daily } \\
\text { homework and was } \\
\text { offered multiple times } \\
\text { during 1994-2013 }\end{array}$ & & \\
\hline $\begin{array}{l}\text { Chilengi et al. } \\
2013 \text { [22] }\end{array}$ & $\begin{array}{l}\text { Web-based } \\
\text { targeting African } \\
\text { researchers }\end{array}$ & $\begin{array}{l}\text { Goal: Complement other } \\
\text { forms of learning though } \\
\text { online training on health } \\
\text { research ethics and good } \\
\text { clinical practice } \\
\text { Competencies: Health } \\
\text { research ethics and good } \\
\text { clinical practice }\end{array}$ & $\begin{array}{l}1155 \text { trainees, } 958 \text { African: } \\
\text { Researchers from multiple } \\
\text { countries }\end{array}$ & $\begin{array}{l}\text { Trainers or practitioners of } \\
\text { research ethics within } \\
\text { Africa }\end{array}$ & Training lasted 100 days & $\begin{array}{l}\text { Total funds: Not reported } \\
\text { (software expenses } \\
\text { mentioned) Funding } \\
\text { source and partnerships: } \\
\text { EDCPT and AMANET }\end{array}$ & No \\
\hline $\begin{array}{l}\text { Harries et al. } \\
2003 \text { [21] }\end{array}$ & Malawi & $\begin{array}{l}\text { Goal: Operational } \\
\text { research training for TB } \\
\text { related research } \\
\text { Competencies: Data } \\
\text { collection and data } \\
\text { analysis, research protocol } \\
\text { and manuscript writing }\end{array}$ & $\begin{array}{l}25 \text { trainees, all African: TB } \\
\text { officers from district and } \\
\text { mission hospitals within } \\
\text { the country }\end{array}$ & $\begin{array}{l}\text { NTP facilitators from the } \\
\text { Central Unit and Regional } \\
\text { TB offices }\end{array}$ & $\begin{array}{l}\text { Training activities lasted } 1 \\
1 / 2 \text { days of seminar on } \\
\text { OR and development of } \\
\text { protocol, } 6 \text { months of } \\
\text { field work, } 1 \text { day } \\
\text { workshop of data analysis } \\
\text { and writing a paper and } \\
\text { was offered once in } 2000\end{array}$ & $\begin{array}{l}\text { Total funds: Not reported } \\
\text { (financial incentives } \\
\text { mentioned) Funding } \\
\text { source and partnerships: } \\
\text { National governments } \\
\text { and District TB units }\end{array}$ & $\begin{array}{l}\text { Field supervisory visits } \\
\text { are carried out once } \\
\text { or twice yearly by } \\
\text { central unit to assess } \\
\text { data collection }\end{array}$ \\
\hline $\begin{array}{l}\text { Laserson et al. } \\
2005 \text { [12] }\end{array}$ & $\begin{array}{l}\text { South Africa } \\
\text { then expanded }\end{array}$ & $\begin{array}{l}\text { Goal: building capacity in } \\
\text { basic epidemiology and }\end{array}$ & $\begin{array}{l}149 \text { of various } \\
\text { nationalities: National, }\end{array}$ & & $\begin{array}{l}\text { Training activities lasted } \\
6 \text { days, involving lectures, }\end{array}$ & $\begin{array}{l}\text { Total funds: US\$2000 - } \\
\text { 20,000 Funding source }\end{array}$ & $\begin{array}{l}\text { Technical assistance is } \\
\text { provided during field }\end{array}$ \\
\hline
\end{tabular}


Table 1 Characteristics of approaches in the review of research capacity strengthening activities in sub-Saharan Africa (Continued)

\begin{tabular}{|c|c|c|c|c|c|c|c|}
\hline & $\begin{array}{l}\text { to regional } \\
\text { course }\end{array}$ & $\begin{array}{l}\text { operations research } \\
\text { Competencies: Qualitative } \\
\text { methods, study design, } \\
\text { data collection and data } \\
\text { analysis, research protocol } \\
\text { and manuscript writing }\end{array}$ & $\begin{array}{l}\text { provincial, and district- } \\
\text { level NTP managers and } \\
\text { TB laboratory directors } \\
\text { and staff }\end{array}$ & $\begin{array}{l}\text { International and in- } \\
\text { country epidemiologists } \\
\text { and TB experts }\end{array}$ & $\begin{array}{l}\text { field exercises, } \\
\text { development of OR } \\
\text { protocol and } 12 \text { months } \\
\text { of field implementation } \\
\text { of the protocol, often in } \\
\text { groups and was offered } \\
\text { nine times during 1997- } \\
2004\end{array}$ & $\begin{array}{l}\text { and partnerships: NTP in } \\
\text { various countries, USAID, } \\
\text { WHO, CDC, Pan American } \\
\text { Health Organization }\end{array}$ & $\begin{array}{l}\text { activities including } \\
\text { further training }\end{array}$ \\
\hline \multirow[t]{2}{*}{$\begin{array}{l}\text { Varkevisser et al. } \\
2001[13]\end{array}$} & \multirow[t]{2}{*}{$\begin{array}{l}\text { Southern African } \\
\text { Region }\end{array}$} & \multirow{2}{*}{$\begin{array}{l}\text { Goal: increase national } \\
\text { capacity for operational } \\
\text { research Competencies: } \\
\text { Data collection and data } \\
\text { analysis, research protocol } \\
\text { and manuscript writing }\end{array}$} & \multirow{2}{*}{$\begin{array}{l}1159 \text { trainees, all African: } \\
\text { Higher and middle level } \\
\text { health workers from } \\
\text { provincial and district } \\
\text { level }\end{array}$} & \multirow{2}{*}{$\begin{array}{l}\text { University staff, senior } \\
\text { health trainers and } \\
\text { higher-level health staff } \\
\text { who have completed an } \\
\text { HSR methodology before }\end{array}$} & \multirow{2}{*}{$\begin{array}{l}\text { Training activities lasted } \\
14-16 \text { days of workshop } \\
\text { to develop research } \\
\text { proposal, } 6 \text { months to } \\
\text { collect data, followed by } \\
12-14 \text { days of data } \\
\text { analysis and writing a } \\
\text { report and was offered } 50 \\
\text { times during 1987-1997 }\end{array}$} & $\begin{array}{l}\text { Total funds: } \\
\text { USD } \$ 5000-\$ 8000 \text { per } \\
\text { study }\end{array}$ & \multirow{2}{*}{$\begin{array}{l}\text { Institutional support } \\
\text { from local authority is } \\
\text { sought through } \\
\text { special meetings at } \\
\text { national and inter- } \\
\text { country level orga- } \\
\text { nized at regular inter- } \\
\text { vals ( } 2-3 \text { years) }\end{array}$} \\
\hline & & & & & & $\begin{array}{l}\text { Funding source and } \\
\text { partnerships: WHO, The } \\
\text { Netherlands Ministry of } \\
\text { Development and } \\
\text { Cooperation, USAID, IDRC, } \\
\text { Norad }\end{array}$ & \\
\hline $\begin{array}{l}\text { Zachariah et al. } \\
2011 \text { [23] }\end{array}$ & Multiple countries & $\begin{array}{l}\text { Goal: building leadership } \\
\text { in operational research } \\
\text { Competencies: Research } \\
\text { questions and protocol } \\
\text { development, data } \\
\text { management and } \\
\text { analysis, paper writing }\end{array}$ & $\begin{array}{l}\text { Number of trainees not } \\
\text { reported: Persons who } \\
\text { work within disease } \\
\text { programmes and who } \\
\text { are committed and have } \\
\text { opportunities to carry out } \\
\text { operational research }\end{array}$ & International & $\begin{array}{l}\text { Training activities lasted } \\
3 \text { weeks spread over } \\
9 \text { months with significant } \\
\text { intervals between } \\
\text { modules and frequency } \\
\text { of offering is not reported }\end{array}$ & $\begin{array}{l}\text { Total funds: } \$ 500-\$ 1500 \\
\text { small grants Funding } \\
\text { source and partnerships: } \\
\text { The Union/MSF }\end{array}$ & $\begin{array}{l}\text { Technical support } \\
\text { throughout the } \\
\text { program }\end{array}$ \\
\hline
\end{tabular}

AMANET African Malaria Network Trust, APHRC African Population and Health Research Center, ATS American Thoracic Society, CDC US Centers for Disease Control, CIBAF Centre Interdisciplinaire de Bioethique pour L'Afrique Francophone, CU University of Colorado-Boulder, DRC Democratic Republic of Congo, EDCPT European Developing Countries Clinical Trials Partnership, GIRIE Groupe Interproject de Reflexion et d'Intervention en Ethique, IDRC International Development Research Centre, IRB Institutional Review Board, JHU Johns Hopkins University, KSPH Kinshasa School of Public Health, M\&E Monitoring and Evaluation, MakSPH Makerere University School of Public Health, MSF Médecins Sans Frontières, NGO Non-Governmental Organization, NIH National Institutes of Health, Norad Norwegian Agency for Development Cooperation, NTP National TB Control Program, OR Reach Out, TB Tuberculosis, UNC University of North Carolina, USAID United States Agency for International Development, WHO World Health Organization 
Table 2 Evaluation details of six trainings with short evaluation periods

\begin{tabular}{|c|c|c|c|c|c|c|c|c|}
\hline \multirow[b]{2}{*}{ Indicators } & & \multicolumn{7}{|c|}{ Short term evaluation period trainings } \\
\hline & & $\begin{array}{l}\text { Tshikala et al. } \\
2012[19]\end{array}$ & $\begin{array}{l}\text { Chilengi et al. } \\
2013 \text { [22] }\end{array}$ & $\begin{array}{l}\text { Mbuagbaw } \\
\text { et al. } 2011[17]\end{array}$ & $\begin{array}{l}\text { Ajuwon and } \\
\text { Kass } 2008[14]\end{array}$ & $\begin{array}{l}\text { Njie-Carr } \\
\text { et al. } 2012 \text { [18] }\end{array}$ & $\begin{array}{l}\text { Harries et al. } \\
{[21] 2003}\end{array}$ & $\begin{array}{l}\text { Percentage of studies } \\
\text { reporting on indicators }\end{array}$ \\
\hline \multicolumn{9}{|l|}{ Study's evaluation approach } \\
\hline \multicolumn{2}{|c|}{ Evaluation framework used } & Not evaluated & NR & NR & NR & & NR & 16.7 \\
\hline \multicolumn{2}{|c|}{ Evaluation method (qualitative/quantitative) } & & Quant & Quant & Quant & Quant & Quant & \\
\hline \multicolumn{2}{|c|}{$\begin{array}{l}\text { Details (satisfaction survey/self-reported } \\
\text { changes/pre-post skills test/research outputs survey) }\end{array}$} & & Test Scores & Satisfaction surveys & Pre/Post Tests & Surveys & Program Data & \\
\hline \multicolumn{2}{|c|}{ Program evaluation period months } & & NR & 0.13 & 1 & 1 & 15 & \\
\hline \multicolumn{9}{|c|}{$\begin{array}{l}\text { Reviews measures of effectiveness of RCS, based on } \\
\text { Cooke's framework }\end{array}$} \\
\hline \multirow[t]{3}{*}{$\begin{array}{l}\text { Improved confidence } \\
\text { and skills }\end{array}$} & $\begin{array}{l}\text { Evidence of knowledge } \\
\text { and skills developed } \\
\text { (e.g., improved post-test } \\
\text { scores) }\end{array}$ & Yes & Yes & Yes & Yes & Yes & Yes & 100.0 \\
\hline & $\begin{array}{l}\text { Evidence of confidence } \\
\text { building (e.g., trainees } \\
\text { become trainers; } \\
\text { obtained research-related } \\
\text { jobs) }\end{array}$ & NR & NR & NR & NR & Yes & NR & 16.7 \\
\hline & $\begin{array}{l}\text { Research undertaken after } \\
\text { training } \\
\text { (e.g., involvement in } \\
\text { subsequent research) }\end{array}$ & NR & NR & NR & NR & NR & NR & 0.0 \\
\hline \multirow[t]{4}{*}{$\begin{array}{l}\text { Research is close to } \\
\text { practice }\end{array}$} & $\begin{array}{l}\text { Practitioner and program } \\
\text { staff involvement } \\
\text { (e.g., nurse, manager } \\
\text { trainees) }\end{array}$ & Yes & No & No & No & Yes & Yes & 50.0 \\
\hline & $\begin{array}{l}\text { Research relevant to or } \\
\text { used in practice } \\
\text { (e.g., reported changes in } \\
\text { practice) }\end{array}$ & Yes & $N R$ & NR & $N R$ & Yes & Yes & 50.0 \\
\hline & $\begin{array}{l}\text { Patient centred outcome } \\
\text { measures used }\end{array}$ & $N R$ & NR & NR & NR & NR & NR & 0.0 \\
\hline & $\begin{array}{l}\text { Action oriented } \\
\text { methodologies used } \\
\text { (e.g., research done on } \\
\text { quality care) }\end{array}$ & $N R$ & $N R$ & NR & $N R$ & NR & Yes & 16.7 \\
\hline \multirow[t]{2}{*}{$\begin{array}{l}\text { Research enhanced by } \\
\text { partnerships }\end{array}$} & $\begin{array}{l}\text { Between novice and } \\
\text { experienced researchers }\end{array}$ & No & NR & No & No & No & No & 0.0 \\
\hline & $\begin{array}{l}\text { Inter-professionals } \\
\text { linkages (e.g., between } \\
\text { researchers, policy }\end{array}$ & Yes & NR & No & Yes & Yes & Yes & 66.7 \\
\hline
\end{tabular}


Table 2 Evaluation details of six trainings with short evaluation periods (Continued)

\begin{tabular}{|c|c|c|c|c|c|c|c|c|}
\hline & $\begin{array}{l}\text { makers, different } \\
\text { disciplines) }\end{array}$ & & & & & & & \\
\hline \multirow[t]{3}{*}{ Impactful dissemination } & $\begin{array}{l}\text { Publications in peer- } \\
\text { reviewed journals }\end{array}$ & No & NR & $N R$ & NR & NR & No & 0.0 \\
\hline & $\begin{array}{l}\text { Conference/workshop } \\
\text { presentation }\end{array}$ & No & NR & NR & NR & NR & NR & 0.0 \\
\hline & $\begin{array}{l}\text { Evidenced of applied } \\
\text { research findings } \\
\text { (e.g., changes in policy/ } \\
\text { practice reported) }\end{array}$ & No & NR & $N R$ & NR & NR & NR & 0.0 \\
\hline \multirow[t]{3}{*}{$\begin{array}{l}\text { Continuity and } \\
\text { sustainability }\end{array}$} & $\begin{array}{l}\text { Successful access of } \\
\text { funding (grants/ } \\
\text { fellowships) }\end{array}$ & No & NR & NR & NR & NR & NR & 0.0 \\
\hline & $\begin{array}{l}\text { Enduring collaborations } \\
\text { (e.g., relationship building } \\
\text { between involved } \\
\text { institutions to promote } \\
\text { individual } \\
\text { training) }\end{array}$ & Yes & NR & NR & NR & NR & NR & 16.7 \\
\hline & $\begin{array}{l}\text { Continued mentorship } \\
\text { and supervision }\end{array}$ & No & NR & NR & NR & Yes & Yes & 33.3 \\
\hline \multirow[t]{4}{*}{$\begin{array}{l}\text { Infrastructure for } \\
\text { research }\end{array}$} & $\begin{array}{l}\text { Institutional support for } \\
\text { undertaking research }\end{array}$ & Yes & NR & NR & NR & NR & Yes & 33.3 \\
\hline & Protected research time & No & NR & $N R$ & NR & NR & NR & 0.0 \\
\hline & Budget line & NR & NR & NR & NR & NR & Yes & 16.7 \\
\hline & $\begin{array}{l}\text { Mentorship and } \\
\text { supervision structures }\end{array}$ & No & NR & NR & NR & NR & NR & 0.0 \\
\hline
\end{tabular}


measures, access to funding post training, availability of protected research time, or existence of mentorship and supervision structures.

\section{Training program with long-term evaluation period}

All training programs reported on an increase in research knowledge and skills and research undertaken after training (100\%; Table 3). More than half of these trainings (50-87.5\%), reported evidence of confidence building among trainees, the involvement of practitioner and program staff in the training, the relevance or use of training-related research in practice, the existence of inter-professional linkages, publications in peer-reviewed journals, evidence of applied research findings, continued mentorship and supervision, and enduring collaborations. None of these training programs either reported on or used availability of protected research time, budget lines, or existence of mentorship and supervision structures.

\section{Challenges, innovations and recommendations}

This review identified major themes regarding challenges to research capacity strengthening activities and suggested corresponding innovations and recommendations to address the challenges (Table 4). Common challenges to capacity strengthening were lack of mentorship and institutional support $[10,13,16,18,20,21,23]$; insufficient time for research activities and drop out $[10,16$, $18,20,21]$; lack of sufficient budget for research activities $[11,13,18,23]$; poor research infrastructure $[12,13$, $17,18,23]$; and difficulty in publishing in international journals [11, 21, 23]; three papers did not report any challenges [16, 18, 21].

Challenges faced by participants are distinguished to those faced by facilitators and organizers. On the one hand, participants who lack support and mentorship from supervisors and managers are more likely to drop out of the training or their research projects are likely to be delayed. On the other hand training organizers and facilitators find it difficult when participants are pulled out of the training because of other work responsibilities, particularly when training organizers and the organization where a participant works do not have a memorandum of understanding. Infrastructural challenges such as poor internet and inadequate space and equipment affect both participants and facilitators' performances. Further, when participants have heavy workloads they are likely to drop out of the training, thus affecting trainers and organizers. A lack of funding implies that any research requiring funds will not be performed and training activities could be hampered, for example, when participants need transport and do not have money. For organizers, a lack of funding could mark the end of training activities since they face shortages of materials, facilitators, and poor infrastructure.

Various recommendations and innovations are proposed to address the challenges to research capacity strengthening. Institutional support and mentorship is achieved in different ways such as provision of mentorship and supervision visits by programme managers $[10,16]$, developing strong professional network [15], and seeking commitment from stakeholders [13, 16, $18]$. Increased time for research $[18,23]$, suitable training schedule [18], and creating web-based training helps to tackle the challenge of insufficient time. Building more funding resources for research activities [11], embedding research into a health program [21], and integrating courses into existing curriculum $[16,20]$ are recommended as strategies to address the lack of funding. The challenge of publication could be addressed through provision of mentorship on publication process [11] and finding other means of dissemination than international journals [21], for example, through special meetings with stakeholders. Provision of further training to improve writing skills of young researchers would increase the likelihood of having a manuscript accepted for publication.

\section{Discussion}

In this systematic review, we identified 14 papers that describe research capacity strengthening activities outside of formal academic programs in sub-Saharan Africa. We found that training programs generally fell into two categories: longer training programs covering multiple competencies and shorter training programs targeting a single research competency. Generally, shorter programs did not have practicum projects as part of the training nor did they provide mentorship/support post-training. These two features make such programs less expensive and less time consuming and therefore more feasible for many settings. However, though their contribution to the increase in research skills and knowledge is recognized, we found little evidence that links these programs to the research projects conducted. Further, offering trainings that focus on narrow competencies would then require multiple trainings to enable participants to take a research question through to publication, if this is the intended goal.

Alternatively, training programs which are more comprehensive yield better outcomes in terms of the number of research projects conducted and resulting publications. They are offered over a longer period and often require ongoing mentorship/support. The demands on both human and financial resources make such trainings more expensive and time consuming and therefore less accessible to many organizations. 
Table 3 Evaluation details of eight trainings with long evaluation periods

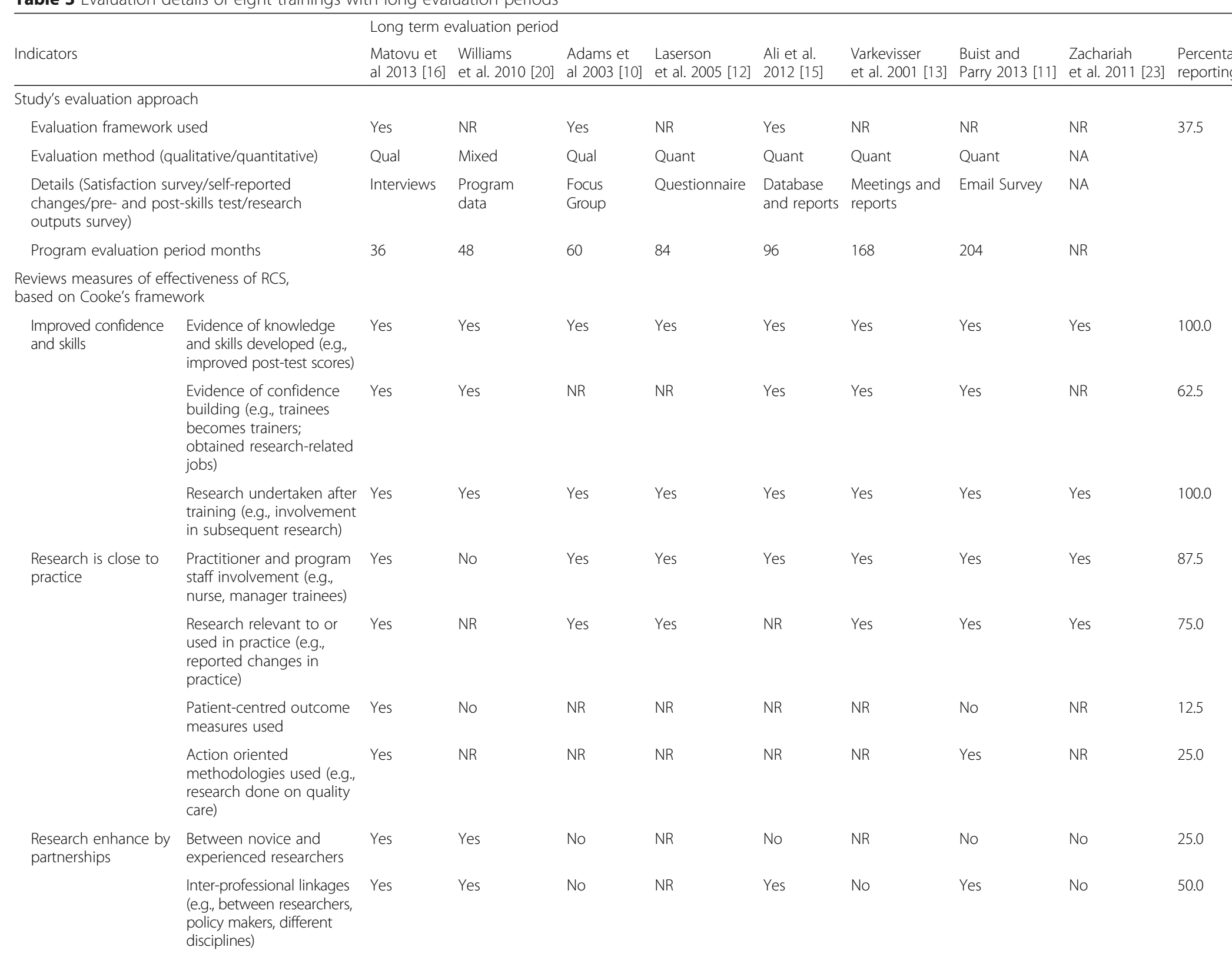


Table 3 Evaluation details of eight trainings with long evaluation periods (Continued)

\begin{tabular}{|c|c|c|c|c|c|c|c|c|c|c|}
\hline \multirow[t]{3}{*}{$\begin{array}{l}\text { Impactful } \\
\text { dissemination }\end{array}$} & $\begin{array}{l}\text { Publications in peer- } \\
\text { reviewed journals }\end{array}$ & $N R$ & $N R$ & NR & $N R$ & Yes & Yes & Yes & Yes & 50.0 \\
\hline & $\begin{array}{l}\text { Conference/workshop } \\
\text { presentation }\end{array}$ & Yes & $N R$ & NR & $N R$ & Yes & $N R$ & Yes & NR & 37.5 \\
\hline & $\begin{array}{l}\text { Evidenced of applied } \\
\text { research findings (e.g., } \\
\text { changes in policy/practice } \\
\text { reported) }\end{array}$ & Yes & $N R$ & NR & Yes & NR & Yes & Yes & Yes & 62.5 \\
\hline \multirow[t]{3}{*}{$\begin{array}{l}\text { Continuity and } \\
\text { sustainability }\end{array}$} & $\begin{array}{l}\text { Successful access of } \\
\text { funding (grants/ } \\
\text { fellowships) }\end{array}$ & NR & $N R$ & NR & $N R$ & Yes & $N R$ & Yes & Yes & 37.5 \\
\hline & $\begin{array}{l}\text { Enduring collaborations } \\
\text { (e.g., relationship building } \\
\text { between involved } \\
\text { institutions to promote } \\
\text { individual training) }\end{array}$ & Yes & Yes & NR & $N R$ & Yes & Yes & Yes & $N R$ & 62.5 \\
\hline & $\begin{array}{l}\text { Continued mentorship } \\
\text { and supervision }\end{array}$ & Yes & $N R$ & No & Yes & Yes & $N R$ & Yes & Yes & 62.5 \\
\hline \multirow[t]{4}{*}{$\begin{array}{l}\text { Infrastructure for } \\
\text { research }\end{array}$} & $\begin{array}{l}\text { Institutional support for } \\
\text { undertaking research }\end{array}$ & Yes & NR & NR & NR & NR & Yes & $N R$ & NR & 25.0 \\
\hline & Protected research time & $N R$ & $N R$ & NR & $N R$ & NR & $N R$ & $N R$ & NR & 0.0 \\
\hline & Budget line & $N R$ & $N R$ & NR & $N R$ & $N R$ & $N R$ & $N R$ & $N R$ & 0.0 \\
\hline & $\begin{array}{l}\text { Mentorship and } \\
\text { supervision structures }\end{array}$ & $N R$ & NR & NR & NR & $N R$ & NR & $N R$ & NR & 0.0 \\
\hline
\end{tabular}


Table 4 Challenges, recommendations and innovations regarding research capacity strengthening activities

Challenge
Lack of mentorship and institutional
support
support

\section{Poor research infrastructure}

Insufficient time for research and program dropouts

Lack of funds for research activities

Difficulty in publishing papers in international journals

As faced by trainees

Participants' initiatives blocked by

managers [10]

Drop out from training program

because of no mentorship $[16,18,20]$

Delay in completing research projects because of no mentorship [16]

Lack of communication between

participants and supervisors [21]

\section{Poor internet [17]}

Inadequate space and lack of equipment $[18,23]$

Trainees get absorbed into routine work and responsibilities [16]

Trainees take jobs with other institutions [16]

Lack of resources to conduct research activities $[11,13,23]$

Difficulty in accessing training

location [18]

Difficulties in publishing in international journals $[11,21,23]$

As faced by organizers/facilitators

nnovations/recommendations

Provide mentorship to participants by

Lack of strategies encouraging recent

trainees to apply new learning within
the services [10] Difficulty getting buy-in from institutions [16, 23]

nagers to enhance application of acquired skills on the job [10]

During application approvals,

organizational commitment to in-service training for capacity development [10]

Weak co-ordination due to incompetency of leaders [13]

Support professional network and alternative communication pathways to

improve intra- and inter-program collaboration [15]

Engage with institutions from the

beginning and get commitment from program leadership [16, 18]

Sensitize policy-makers and health managers through special meetings [13]

\section{Poor internet [17]}

Difficulty in securing adequate space for research activities $[12,13,18,23]$

Loss of trainees through dropout $[16,18$ 20]

Trainers do not have resources nor authority to conduct effective follow-up within workplace [10] Mismatches between participants' capabilities and training priorities [21]

Dependence on external institutions or donors for funding [13]

Improve internet access [17, 23]

Provide budget lines dedicated for improving research infrastructure [23]

Conducting training activities at the workplace

Increase time allocated to research activities [18, 23]

Suitable training schedule [18]

Establish strong selection criteria to minimize dropouts [23]

Add distance learning to face-to-face classes

Provide support supervision to trainees by program staff and/or mentors [16] Build more resources for funding [11]

Embed research agenda into health program [21]

Develop strong institutional infrastructure (administrative leadership) [18]

Integrate courses into existing curriculum $[16,20]$

Mentor on publication process [11]

Strengthen selection criteria to get strong candidates 
Language barriers and differences in educational levels
Trainees face communication

challenges [18]
Difficult to manage a group of different levels of education [21] and/or speaking different languages [18]

Explore other opportunities such as

publishing in local journals and

presenting at local meetings [21]

Provide further training [21]

Strategic groupings of participants with similar skill levels [21] 
Most of the studies in our review did not report on the program implementation costs. When reported, these costs varied widely, between $\$ 500$ and $\$ 20,000$ per project, depending on scope of the project, location, and duration of training. While actual costing of programs is difficult, reporting of the estimated expenditure are important to other people planning these training activities, particularly because resource allocation is among the major barriers in research capacity strengthening activities. Except for one program that was a national program [21], programs primarily relied on North-South partnerships for funding, highlighting the need for strengthening partnerships with more focus on South research agenda [24], as well as galvanizing national resources and increasing South-South research collaboration.

In addition to variability in the program approaches, there was a large variability in evaluation approaches. Self-report surveys, pre-/post-tests, interviews, email questionnaires, and system approaches were all found to have been used. Self-report surveys and pre-/post-tests were used by shorter training programs and administered during or shortly after the completion of training. That period was not enough for such training to have had an impact on participants, but rather they reported on the perception of participants about the course and whether changes in knowledge have occurred. Longer trainings, on the other hand, were more likely to followup participants through implementation of research projects over which additional technical assistance and mentorship are provided. Specific deliverables for most of those training programs which include writing a protocol and/or writing and publishing a manuscript enable them to determine the level of their success. Understandably, the long period of implementation, in addition to both technical and financial support provided to complete research projects, is likely to increase the number of protocols written, research projects conducted and published, and the influence in policy and practice change among others. However, much needs to be done to fully understand the impact of such capacity strengthening trainings. For instance, better baseline assessment using comprehensive tools, such as those employed by systems approach [25], are needed as well as better reporting on whether there were other outside enabling factors.

The evaluation metrics for research capacity strengthening programs are debated in the literature. Some suggest that success should be measured in terms of papers published [26]; however, this implies that writing a paper is the ultimate goal for the training or target competency desired by the individual. Others advise that change in policy and practice should be the end goal of research capacity strengthening activities in order to improve the quality of service delivery [27]. There are few training programs that cover all necessary competencies to write and publish a research paper as an indicator of success; this requires not only substantial resources in terms of trainers and mentors, time, and money, but also strong candidates, thus limiting the number of training participants. Further, using research to change policy is difficult, requiring ongoing engagement and co-operation between all stakeholders, and documenting such change in a concrete and objective way is even more challenging. Alternatively, Harries et al. [21] advocates for embedding research training activities into existing health programs. This suggests that training is budgeted for as any other activity of the program and often times participants in that training are staff who work within health programs.

The challenges to research capacity strengthening identified in this review have been observed by others. Several studies report limited funding for research $[6,8$, $26,28,29]$, no dedicated time for research [3, 26, 29], and a lack of mentorship and institutional support [8, 13, 27]. In addition, challenges identified but not discussed in papers in this review include difficulties in carrying out quality evaluation particularly for long term outcomes and the imbalanced focus on research methods and process at the expense of research advocacy, promotion, negotiation, and resource mobilization [30]. These challenges are complex and call for sustainable partnerships and commitment to the goals of research capacity strengthening in Africa.

While academic and non-academic training programs face similar challenges, some of the challenges, such as lack of institutional support or research leadership, are more pronounced in non-academic settings. Our review identified one program with institutional support [13] which also had the most significant and quantified impact on society through policy and practice changes. We believe that the research developed as part of academic trainings is more likely to be published because of the existence of such support. Furthermore, trainees in academic programs tend to have time separated out for research and thus do not face the similar challenge of balancing work and research training concurrently. Academic programs may also be appealing because of the existing accreditation process that is difficult for the non-academic program.

There were two primary limitations to this systematic review. First, for the articles identified, relevant information on important features, including features that would support replicability, were missing. For example, it is possible that some programs offered on-going mentorship, but we were unable to report this feature because it was not described in the paper. Information on financial and material resources, qualifications, and number 
of trainers/facilitators needed to undertake capacity strengthening activities were poorly reported, which not only is a limitation of this review, but may weaken the ability to replicate the program in other settings. A second limitation is that this review only included scientific articles that had the abstract and full paper available in English. Therefore, we believe that programs published in languages other than English or presenting their results in grey literature may have been overlooked. Though grey literature may offer more detailed information about training programs, their use is hampered by the difficulty in accessing reports years after their production and limited information on the individuals involved in producing the report. However, because of publication bias in scientific literature, this review may have missed training programs that were deemed less successful, less "innovative", or may have had less academic collaboration. On the other hand, the limited number of articles and the limited detail in the articles serves as a call-to-action for individuals developing and leading such research capacity strengthening activities to ensure that approaches and lessons learnt are shared more widely and with enough details to facilitate the replication of their activities in other settings.

\section{Conclusion}

Research capacity strengthening activities through nonacademic trainings can generate researchers capable of developing research question through to publication and integration of findings into policy and practice. Institutional support, increased funds, and dedicated time for research activities are critical factors that lead to development of successful health research capacity strengthening programs. Achieving representation of African authors in scientific health literature may rely in part on the outcome of research capacity strengthening programs. However, few publications examine this in a robust way or with sufficient detail for replication. Replication of successful models relies on robust evaluation methods and program documentation made accessible in the peer-reviewed literature. We thus recommend further research into feasible methods of tracking medium term and long term research impact. Further, future reviews could explore research capacity strengthening trainings in other regions.

\section{Competing interests}

The authors declare that they have no competing interests.

\section{Authors' contributions}

LM led all aspects of this paper including study design, data collection, data analysis, and preparation of manuscript. DR supported all aspects of this paper from study design, data collection, and data analysis, provided critical review to improve the paper, and approved the final version. JO supported data collection and critically reviewed the manuscript for improvement and approved the final version. MPN provided technical support to data collection and data analysis and contributed to paper editing for improvement and approved the final version. CA, CW, PB, and LH supported interpretation of findings and reviewed and edited the paper and approved the final version. BHG provided direct supervision, mentorship, and technical support to LM from the beginning until the completion of the manuscript and approved the final version.

\section{Acknowledgements}

LM conducted this study as part of his Global Health Corps Fellowship at Partners In Health/Inshuti Mu Buzima. BHG received support from the Department of Global Health and Social Medicine Research Core at Harvard Medical School.

\section{Author details}

${ }^{1}$ Partners In Health-Inshuti Mu Buzima, P.O. Box 3432, Kigali, Rwanda. ${ }^{2}$ Global Health Corps, One Penn Plaza, Suite 6271, New York, NY 10119, USA.

'Department of Global Health and Social Medicine, Harvard Medical School, 641 Huntington Avenue, Boston, MA 02115, USA.

Received: 1 August 2014 Accepted: 25 May 2015

\section{References}

1. Bates I, Taegtmeyer M, Squire SB, Ansong D, Nhlema-Simwaka B, Baba A, et al. Indicators of sustainable capacity building for health research: analysis of four African case studies. Health Res Policy Syst. 2011;9:14.

2. Nachega JB, Uthman OA, Ho Y-S, Lo M, Anude C, Kayembe P, et al. Current status and future prospects of epidemiology and public health training and research in the WHO African region. Int J Epidemiol. 2012;41:1829-46.

3. Zachariah R, Harries AD, Ishikawa N, Rieder HL, Bissell K, Laserson K, et al. Operational research in low-income countries: what, why, and how? Lancet Infect Dis. 2009;9:711-7.

4. Kabiru CW, Izugbara CO, Wambugu SW, Ezeh AC. Capacity development for health research in Africa: experiences managing the African doctoral dissertation research fellowship program. Health Res Policy Syst. 2010;8:21.

5. Adam T, Ahmad S, Bigdeli M, Ghaffar A, Røttingen J-A. Trends in health policy and systems research over the past decade: still too little capacity in low-income countries. PLoS One. 2011;6, e27263.

6. Sitthi-Amorn C, Somrongthong R. Strengthening health research capacity in developing countries: a critical element for achieving health equity. BMJ. 2000;321:813-7.

7. Minja H, Nsanzabana C, Maure C, Hoffmann A, Rumisha S, Ogundahunsi O, et al. Impact of health research capacity strengthening in low- and middle-income countries: the case of WHO/TDR programmes. PLoS Negl Trop Dis. 2011;5, e1351.

8. Lansang MA, Dennis R. Building capacity in health research in the developing world. Bull World Health Organ. 2004;82:764-70.

9. Cooke J. A framework to evaluate research capacity building in health care. BMC Fam Pract. 2005;11:1-11

10. Adams J, Schaffer A, Lewin S, Zwarenstein M, van der Walt H. Health systems research training enhances workplace research skills: a qualitative evaluation. J Contin Educ Health Prof. 2003;23:210-20.

11. Buist AS, Parry V. The American thoracic society methods in epidemiologic, clinical, and operations research program. A research capacity-building program in low- and middle-income countries. Ann Am Thorac Soc. 2013;10:281-9.

12. Laserson KF, Binkin NJ, Thorpe LE, Laing R, lademarco MF, Bloom A, et al. Capacity building for international tuberculosis control through operations research training. Int J Tuberc Lung Dis. 2005;9(April 2004):145-50.

13. Varkevisser CM, Mwaluko GM, Le Grand A. Research in action: the training approach of the joint health systems research project for the Southern African region. Health Policy Plan. 2001;16:281-91.

14. Ajuwon AJ, Kass N. Outcome of a research ethics training workshop among clinicians and scientists in a Nigerian University. BMC Med Ethics. 2008;9:1.

15. Ali J, Hyder AA, Kass NE. Research ethics capacity development in Africa: exploring a model for individual success. Dev World Bioeth. 2012;12:55-62

16. Matovu JKB, Wanyenze RK, Mawemuko S, Okui O, Bazeyo W, Serwadda D. Strengthening health workforce capacity through work-based training. BMC Int Health Hum Rights. 2013;13:8.

17. Mbuagbaw L, Wiysonge CS, Nsagha DS, Ongolo-Zogo P, Pantoja T. An introduction to systematic reviews and meta-analysis: a workshop report on 
promoting evidence based medical practice through capacity building in research synthesis. Pan Afr Med J. 2011;8:15.

18. Njie-Carr V, Kalengé S, Kelley J, Wilson A, Muliira JK, Nabirye RC, et al. Research capacity-building program for clinicians and staff at a community-based HIV clinic in Uganda: a pre/post evaluation. J Assoc Nurses AIDS Care. 2012;23:431-41.

19. Tshikala T, Mupenda B, Dimany P, Malonga A, llunga V, Rennie S. Engaging with research ethics in central Francophone Africa: reflections on a workshop about ancillary care. Philos Ethics Humanit Med. 2012;7:10.

20. Williams JR, Schatz EJ, Clark BD, Collinson MA, Clark SJ, Menken J, et al. Improving public health training and research capacity in Africa: a replicable model for linking training to health and socio-demographic surveillance data. Glob Health Action. 2010;3.

21. Harries AD, Kemp JR, Salaniponi FM. Developing operational research capacity in hospital tuberculosis control officers in Malawi. Int J Tuberc lung Dis. 2003;7(October 2002):266-70.

22. Chilengi R, Nyika A, Tangwa GB, Noor RA, Ramadhani SW, Bosomprah S, et al. Role of E-learning in teaching health research ethics and good clinical practice in Africa and beyond. Bioeth Educ. 2013;22:110-9.

23. Zachariah R, Reid T, Srinath S, Chakaya J, Legins K, Karunakara U, et al. Building leadership capacity and future leaders in operational research in low-income countries: why and how ? Int J Tuberc Lung Dis. 2011;15:1426-35.

24. Barrett AM, Crossley M, Dachi HA. International collaboration and research capacity building: learning from the EdQual experience. Comp Educ. 2011;47(May):25-43.

25. Hyder AA, Harrison RA, Kass N, Maman S. A case study of research ethics capacity development in Africa. Acad Med. 2007;82:675-83.

26. Zachariah R, Tayler-Smith K, Ngamvithayapong-Yanai J, Ota M, Murakami K, Ohkado A, et al. The published research paper: is it an important indicator of successful operational research at programme level? Trop Med Int Heal. 2010;15:1274-7.

27. Zachariah R, Ford N, Maher D, Bissell K, Van Den BR, Van Den BW, et al. Is operational research delivering the goods? The journey to success in low-income countries. Lancet Infect Dis. 2012;3099:1-7.

28. Laabes EP, Desai R, Zawedde SM, Glew RH. How much longer will Africa have to depend on western nations for support of its capacity-building efforts for biomedical research? Trop Med Int Health. 2011:16:258-62.

29. Stein J, Lewin S, Fairall L, Mayers P, English R, Bheekie A, et al. Building capacity for antiretroviral delivery in South Africa: a qualitative evaluation of the PALSA PLUS nurse training programme. BMC Health Serv Res. 2008;8:240.

30. Cole DC, Boyd A, Aslanyan G, Bates I. Indicators for tracking programmes to strengthen health research capacity in lower- and middle- income countries: a qualitative synthesis. Health Res Policy Syst. 2014;12:1-13.

\section{Submit your next manuscript to BioMed Central and take full advantage of:}

- Convenient online submission

- Thorough peer review

- No space constraints or color figure charges

- Immediate publication on acceptance

- Inclusion in PubMed, CAS, Scopus and Google Scholar

- Research which is freely available for redistribution 